\title{
Management of Malaria in Pregnant Women at the Maternity Unit of the University Teaching Hospital Yalgado Ouedraogo (UTH-YO) in Ouagadougou: About Report on the Quality of Care
}

\author{
Ouattara Adama1,2*, Ouédraogo Smaila1,2, Lankoandé Bako Coulibaly Natacha ${ }^{3}$, \\ Ouédraogo Marie Charlemagne ${ }^{1,2}$, Ouédraogo Ali1,2, Thieba Bonané Blandine ${ }^{1,2}$ \\ ${ }^{1}$ Training and Research Unit in Health Sciences (TRU/HS), University Ouaga 1 Pr Joseph KI ZERBO, Ouagadougou, Burkina Faso \\ ${ }^{2}$ Department of Gynecology and Obstetrics, The University Teaching Hospital Yalgado Ouedraogo of Ouagadougou (UTH-YO), \\ Ouagadougou, Burkina Faso \\ ${ }^{3}$ Department of Gynecology and Obstetrics, The Bogodogo University Teaching Hospital of Ouagadougou (UTH-B), Ouagadougou, \\ Burkina Faso \\ Email: *ouattzangaadama@yahoo.fr
}

\begin{abstract}
How to cite this paper: Adama, O., Smaila, O., Natacha, L.B.C., Charlemagne, O.M., Ali, O. and Blandine, T.B. (2018) Management of Malaria in Pregnant Women at the Maternity Unit of the University Teaching Hospital Yalgado Ouedraogo (UTH-YO) in Ouagadougou: About Report on the Quality of Care. Open Journal of Obstetrics and Gynecology, 8, 1337-1344.

https://doi.org/10.4236/ojog.2018.813135
\end{abstract}

Received: October 6, 2018

Accepted: November 5, 2018

Published: November 8, 2018

Copyright ( 2018 by authors and Scientific Research Publishing Inc. This work is licensed under the Creative Commons Attribution International License (CC BY 4.0).

http://creativecommons.org/licenses/by/4.0/

Open Access

\begin{abstract}
Objective: To do a report on the quality of the treatment of malaria in pregnant women at University Teaching Hospital Yalgado Ouedraogo (UTH-YO) in Ouagadougou. Materials and Methods: This was a descriptive cross-sectional study conducted from 29 July to 02 October 2016 . The targets were pregnant women and gynecology and obstetrics service care providers. The study used an individual questionnaire administered and clinical parasitological research by the Rapid Diagnostic Test (RDT). Some information was verified in the patient's prenatal consultation logbook. A total of 351 women and 44 providers participated in the study. The data was captured on a microcomputer and analyzed using Epi info version 7 software. Results: Regarding preventive measures, patients' responses were correct for long-lasting impregnated mosquito net (LLIN) in $98.8 \%$ of cases and for intermittent preventive treatment (IPT) in $96.5 \%$ of cases. About providers, they had given a good answer in all cases for using LLIN and taking IPT. Regarding care providers' knowledge of the clinical signs of malaria, fever was cited in $95.5 \%$ of cases for simple malaria and Frankish jaundice in $97.7 \%$ of cases for severe malaria. With regard to curative treatment, $77.3 \%$ of the care providers surveyed stated that quinine was the reference molecule for the treatment of simple malaria in pregnant
\end{abstract}


women. In terms of the availability of inputs used for malaria management, our survey found no RDTs. Also, the only molecule available was quinine in its compressed and injectable forms. Conclusion: Care Providers' and patients' knowledge of malaria in the obstetrics and genecology department is quite satisfactory. However, some shortcomings were noted in the provision of screening and curative and preventive treatment. The elimination of malaria in our country will require joint efforts at all levels.

\section{Keywords}

Malaria, Pregnancy, Treatment, UTH-YO

\section{Introduction}

Malaria is an endemo-epidemic, tropical and intertropical parasitic disease caused by hematozoans of the genus Plasmodium, transmitted by mosquitoes of the genus Anopheles [1]. According to the World Health Organization (WHO), there are approximately 10,000 maternal deaths related to this pandemic each year [2]. In Burkina Faso, it is said to be the main reason for consultations, hospitalization and deaths in health facilities. Indeed, in 2013, malaria accounted for $46.5 \%$ of the reasons for consultation, $61.5 \%$ of hospitalizations and $30.5 \%$ of deaths affecting the entire population [3].

To provide an effective response to this pandemic in pregnant women, WHO recommends as priority interventions the effective management of confirmed cases and the adoption of preventive measures, including [4]:

- the use of long-lasting insecticidal nets (LLINs),

- intermittent preventive treatment (IPT),

- rapid diagnosis based on the search for plasmodies either by a thick drop or by a rapid diagnostic test (RDT).

Despite the adoption of these measures at the national level, there is no denying the persistence of morbidity and mortality due to malaria infection [5]. Also, through this study we propose to do a report on the quality on the management of the pandemic at the maternity of Yalgado Ouédraogo University Teaching Hospital (UTH-YO) which is the largest gynecological and obstetrical reference structure in Burkina Faso.

\section{Patients and Method}

This was a descriptive cross-sectional study conducted from July 29 to October 02, 2016, a period of 3 months. The study was conducted in the Department of Gynecology and Obstetrics of Yalgado Ouedraogo University Teaching Hospital (UTH-YO), recognized as a reference structure for regional hospitals and medical centers with surgical antenna of the city of Ouagadougou. This structure serves as a training framework for doctors in specialization, medical and pharmaceutical students, senior health technicians at the University of Ouagadougou 
and students at the National School of Public Health (NSPH) and some private schools. It is equipped with 95 hospital beds. The sampling was opportunistic for all the patients and care givers. Our study had 2 targets:

- as main targets, all pregnant women admitted for inpatient management who have consented to participate in the study,

- as secondary targets, all service care providers who agreed to participate in the study. These providers included doctors, medical students, and midwives.

Data collection used 3 techniques:

- Rapid Diagnostic Test (RDT): A capillary blood sample from $5 \mu$ using a calibrated loop was used to perform the SD Bioline Malaria Antigen Pf test. This is a test for the detection of Plasmodium falciparum HRP2 antigen based on immunochromatography on a strip [4].

- Direct observation of patients and resources available in the department.

- Semi-structured individual interviews with patients and care providers.

After the RDT was performed, each patient was subjected to an individual questionnaire which was completed using the prenatal consultation logbook as well as the patient's record and direct observation of the patient. The data was coded and captured on a microcomputer. The cleaning and analysis was carried out using Epi info software version 7.

\section{Results}

\subsection{Malaria Prevalence}

Of the 351 patients screened, 310 (88.3\%) tested negative and 41 tested positive for RDT. The prevalence of malaria was therefore 11.7 per cent.

\subsection{Characteristics of the Sample Studied}

The average age of the patients was $26.8 \pm 6$ years [13 - 45]. They lived in union in 315 cases $(89.7 \%)$ and alone in 36 cases $(10.3 \%)$. In our study population, $67.6 \%$ were educated. Unemployed patients numbered 218 (62.1\%). They were in the third trimester of their pregnancy in 293 cases (84\%), in the second trimester in 47 cases (13.39\%), in the first trimester in 11 cases (3\%).

There were 44 care givers. Their average age was $33.25 \pm 6.46$ years with extremes ranging from 26 to 55 years. The average years of service was $7.77 \pm 5.02$ years with extremes ranging from 1 to 30 years. There were 24 men (54.55\%) and 20 women (45.45\%).

\subsection{Providers' Knowledge of the Signs of Malaria}

\subsubsection{Simple Malaria}

The distribution of the care providers' knowledge according to their responses on signs of simple malaria was presented in Table 1.

\subsubsection{Severe Malaria}

The distribution of the care providers' knowledge according to their responses on clinical signs of severe malaria was presented in Table 2. 
Table 1. Distribution of care providers' knowledge according to their responses on signs of simple malaria.

\begin{tabular}{ccc}
\hline Signs & Number & Proportion \\
\hline Fever & 42 & 95.5 \\
Headache & 40 & 90.9 \\
Vomiting & 37 & 84 \\
Abdominal pain & 22 & 50 \\
Curves & 30 & 68.2 \\
Joint pain & 29 & 65.9 \\
Diffuse myalgias & 16 & 36.4 \\
Shivers & 32 & 72.7 \\
Myalgias & 5 & 11.4 \\
Asthenia & 27 & 61.4 \\
Arthralgia & 14 & 31.8 \\
\hline
\end{tabular}

Table 2. Distribution of care providers' knowledge according to their responses on clinical signs of severe malaria.

\begin{tabular}{ccc}
\hline Signs & Number & Proportion \\
\hline Frankish jaundice & 43 & 97.7 \\
Prostration & 35 & 79.5 \\
Repeated seizures & 29 & 65.9 \\
Respiratory distress & 18 & 40.9 \\
Acute pulmonary edema & 21 & 47.8 \\
Oligo-anuria & 7 & 15.9 \\
Cardiovascular collapse & 11 & 25 \\
\hline
\end{tabular}

\subsubsection{Biological Signs of Severe Malaria}

The distribution of providers' knowledge according to responses on biological signs of severe malaria has been presented in Table 3.

Anemia (hemoglobin $<5 \mathrm{~g} / \mathrm{dl}$ ) was cited by all providers as the primary biological sign of severe malaria.

\subsection{Providers' Knowledge of Malaria Prevention Measures}

All providers interviewed stated that the use of LLIN and IPT were the primary means of preventing malaria during pregnancy. In addition, they all said that every pregnant woman should sleep under LLIN from the diagnosis of pregnancy until delivery. Sanitation of the living environment as another means of prevention was cited by 06 providers $(13.6 \%)$ and for $4(9.1 \%)$, it was the use of mosquito repellent creams. With regard to taking IPT, $43 \%$ or $97.72 \%$ of providers said that the first dose should be administered in the second trimester and the second in the third trimester, and for $0.1 \%$ or $2.27 \%$, taking should begin as soon as the pregnancy is diagnosed. 
Table 3. Distribution of care providers' knowledge according to their responses on biological signs of severe malaria.

\begin{tabular}{ccc}
\hline Signs & Number & Proportion \\
\hline Anemia $($ hemoglobin level $<5 \mathrm{~g} / \mathrm{dl})$ & 44 & 100 \\
Hyperparasitemia $(>4 \%)$ & 39 & 88.7 \\
Hypoglycemia $(<2 \mathrm{mmol} / \mathrm{L})$ & 41 & 93.3 \\
\hline
\end{tabular}

\subsection{Providers' Knowledge of Curative Treatment of Malaria}

\subsubsection{Simple Malaria}

A total of 34 providers, or $77.3 \%$ of the providers, stated that quinine was the reference molecule for treatment in pregnant women. For $37 \%$ or $84.1 \%$ of providers, the Artemisinin-based Combination treatment (ACT) could be used in pregnant women after the 16th week of amenorrhea. This figure nevertheless indicates how this disease continues to weigh heavily on the health of the population of Burkina Faso.

\subsubsection{Severe Malaria}

For the treatment of severe malaria, $37 \%$ or $84.1 \%$ said that artemether injection was the most recommended form. For $0.4 \%$ or $9.1 \%$ it was injectable artesunate and for $3 \%$ or $6.8 \%$ it was injectable quinine. Regarding the dosage of the molecules, 30 providers, $81.1 \%$, gave a correct dosage for artemether injection, $75 \%$ for artesunate injection and $100 \%$ for quinine injection.

\subsection{Availability of Means of Malaria Management for Pregnant Women in the Ward}

During our investigation, at the gynecology and obstetrics department's drug depot, we observed the absence of IPT, LLIN, and RDT. Only one molecule was available. It was quinine in its injectable form $(400 \mathrm{mg})$ and in its compressed form (300 mg).

\subsection{Pregnant Women's Knowledge about Malaria Prevention}

They relate to the use of the LLIN, TPI, environmental sanitation and the use of mosquito repellent creams. The distribution of patients according to their knowledge of prevention methods was presented in Table 4.

\section{Discussion}

\subsection{Prevalence of Malaria Infection}

The prevalence of malaria infection in the study sample was 11.7\%. Yakpa [6] reported a $35.9 \%$ higher prevalence of malaria infection in pregnant women in her study at Lomé University Hospital. During our investigation, the analysis of the information contained in the health record allowed us to know that some patients had received antimalarial treatment in the weeks preceding their admission to the service. This could have had an impact on the prevalence of infection. 
Table 4. Distribution of patients according to their knowledge about malaria prevention during pregnancy.

\begin{tabular}{ccc}
\hline Means of prevention & Number & Proportion \\
\hline LLIN & 334 & 98.8 \\
IPT & 192 & 96.5 \\
Environmental remediation & 26 & 70.3 \\
Mosquito repellent creams & 63 & 88.8 \\
\hline
\end{tabular}

\subsection{Providers' Knowledge of Clinical Signs of Malaria}

In the providers' assessment of knowledge of the clinical signs of simple malaria, fever was cited by $95.5 \%$ of providers, headache by $90.9 \%$, vomiting by $84.1 \%$, chills by $72.7 \%$, soreness by $68.2 \%$ and asthenia by $61.4 \%$. Feller-Danssokho and al [7] in Senegal also reported similar results.

The National Malaria Control Program (NMCP) defines simple malaria as a fever (axillary temperature greater than or equal to $37.5^{\circ} \mathrm{C}$ ) or a history of a warm body in the last 72 hours and the detection of plasmodium in the blood by a positive rapid diagnostic test (RDT) [8]. Unfortunately, we found no RDT in the service depository. In this context, providers used the syndromic approach in the diagnosis of simple malaria. This implies from the outset a risk of overdiagnostic error with the consequence that antimalarial drugs are misused, which would encourage possible resistance of plasmodium to the molecules used. In addition, the syndromic approach could prolong the patient's hospital stay for other causes of fever. It would therefore be essential to make RDTs available at the maternity ward so that providers can confirm all malaria cases before malaria treatment is started.

For severe malaria, providers were also familiar with clinical signs. Frankish jaundice was cited by $97.7 \%$ of providers, prostration by $79.5 \%$, repeated convulsions by $65.9 \%$, respiratory distress by $40.9 \%$ and acute pulmonary edema by $47.7 \%$. Our results were close to those of Ndiaye [9] in Senegal and Doudou [10] in Côte d'Ivoire. Knowledge of the signs of malaria severity helps to improve patient care [11].

\subsection{Providers' Knowledge of Malaria Prevention}

In our series, all providers had a good knowledge of how to prevent malaria during pregnancy. Indeed, they all stated that the use of LLIN and the use of IPT were the primary means of prevention recommended by the NMCP. In addition, they all agreed that every pregnant woman should sleep on LLIN from the diagnosis of pregnancy until delivery. Sanitation of the living environment as another means of prevention was cited by $13.64 \%$ of providers and for $9.1 \%$, it was the use of mosquito repellent creams. This satisfactory level of providers' knowledge of prevention could be explained by the multitude of training and guidelines received on the subject. 
Regarding IPT use, $97.7 \%$ of providers said that the first dose should be given in the second trimester and the second in the third trimester and $2.2 \%$ said that it should start as soon as the pregnancy is diagnosed. The NMCP recommends that IPT begin in the second trimester of pregnancy and consists of three tablets taken orally each month until delivery with a minimum interval of one month between doses [8]. Regular training targeting the staff of referral centres on the protocol of preventive treatment of malaria in pregnant women is necessary to harmonize knowledge on this very important aspect of management.

\subsection{Providers' Knowledge of the Treatment of Simple Malaria}

More than three quarters $(77.27 \%)$ of the providers surveyed said that quinine was the reference molecule for treatment in pregnant women. For 84.09\%, ACTs could be used after the 16th week of amenorrhea. In the study by Monbrison and al [12] in $2008,68.2 \%$ and $78.2 \%$ of the providers interviewed had recommended quinine and ACTs respectively for the treatment of malaria after the appearance of active fetal movements. Our results could be explained on the one hand by a good dissemination of national guidelines for the treatment of simple malaria and on the other hand, by the existence of a therapeutic protocol in the service. However, $15.91 \%$ of claimants surveyed.

\subsection{Knowledge about the Treatment of Severe Malaria}

Our study revealed that artemether injection was cited by $84.1 \%$ of providers, then artesunate injection by $9.1 \%$ and finally quinine injection by $6.82 \%$ as molecules used for the treatment of severe malaria. This reflects a satisfactory level of knowledge among providers about the treatment of severe malaria. In the national protocol, artesunate injection should be used first-line, then artemether injection and finally quinine injection. The first-line use of artesunate has the advantage of being used intramuscularly and intravenously depending on the urgency. This molecule induces fewer side effects compared to artemether. The latter should be administered exclusively intramuscularly [8] [13].

\section{Conclusion}

Providers' and patients' knowledge of malaria in the obstetrics and gynecology department is quite satisfactory. However, some shortcomings were noted in the provision of screening and curative and preventive treatment. The elimination of malaria in our country will require joint efforts at all levels.

\section{Conflicts of Interest}

The authors declare no conflicts of interest regarding the publication of this paper.

\section{References}

[1] Sirima, S.B., Cotte, A.H., Konaté, A., Moran, A.C., Asamoa, K., Bougouma, E.C., 
Diarra, A., Ouédraogo, A., Parise, M.E. and Newman, R.D. (2006) Malaria Prevention during Pregnancy: Assessing the Disease Burden One Year after Implementation a Program of Intermittent Preventive Treatment in Koupéla District, Burkina Faso. American Journal of Tropical Medicine and Hygiene, 75, 205-211. https://doi.org/10.4269/ajtmh.2006.75.205

[2] WHO (2010) World Malaria Report. World Health Organization, Geneva.

[3] Danis, M. (1991) Symptomatologie du paludisme: In paludisme. Ellipses/AUPELF éd., Paris, 87-89.

[4] WHO (2004) A Strategic Framework for Malaria Prevention and Control during Pregnancy in the African Region. World Health Organization Regional Office for Africa, Brazzaville.

[5] National Malaria Control Programme (NMCP) (2009) Direction de lutte contre la maladie. Ministry of Health. Report of the Household Survey on Malaria in Burkina Faso, 75 p.

[6] Yakpa, J. (2014) Malaria and Pregnancy Seasonality and Relationship with Anaemia and Low Birth Weight in Togo. Master Thesis, University of Lomé, Lomé, 72 p.

[7] Feller-Dansokho, E., Ki-Zerbo, G. and Badiane, S. (1994) Diagnostic and Therapeutic Management of Simple Malaria in the Dakar Region, Senegal. Annales de la Societe belge de medecine tropicale, 74, 291-300.

[8] National Malaria Control Programme (PNLP) (2014) Guidelines nationales pour la prise en charge du paludisme dans les formations sanitaires du Burkina Faso. Ministry of Health. Burkina Faso, 17 p.

[9] Ndiaye, J.L. and Gaye, A. (2001) Infection of the placenta by Plasmodium falciparum in urban areas in Senegal. 6th International Francophone Congress of Tropical Medicine "Health and Urbanization in Africa", Dakar, October 2001, Manuscript No DK/12.2003.

[10] Blanket, T. (2008) Survey on Malaria Knowledge, Attitudes and Practices in Nineteen Health Districts in Côte d'Ivoire Centre de Recherche pour le Développement. Abidjan, $71 \mathrm{p}$.

[11] Ehijie. F.O.E., Augustine, O. and Okpere, E.E. (2007) A Survey of Knowledge, Attitude and Practice of Malaria Management among Pregnant Women from Two Health Cara Facilities in Nigeria. Acta Obstetricia et Gynecologica Scandinavica, 86, 33-36. https://doi.org/10.1080/00016340600984670

[12] Monbrison, F. and Oiege ville, M. (2008) Enseignement de parasitologie.-Association française des enseignants de parasitologie et de mycologie, 3rd Edition. http://anofel.net/

[13] Mohamed, M.O.M. (2008) Etude de la situation de prévention du paludisme chez la femme enceinte: enquête dans les formations sanitaires publiques de la commune de Ouagadougou. Burkina Faso. Master Thesis, Université de Ouagadougou, Ouagadougou, $92 \mathrm{p}$. 